\title{
Feasibility and Acceptability of Utilizing a Smartphone Based Application to Monitor Outpatient Discharge Instruction Compliance in Cardiac Disease Patients around Discharge from Hospitalization
}

\author{
Aimee M. Layton, ${ }^{1}$ James Whitworth, ${ }^{2}$ James Peacock, ${ }^{3}$ Matthew N. Bartels, ${ }^{4}$ \\ Patricia A. Jellen, ${ }^{5}$ and Byron M. Thomashow ${ }^{1}$ \\ ${ }^{1}$ Division of Pulmonary, Allergy and Critical Care Medicine, Department of Medicine, Columbia University Medical Center, \\ VC3-365 Center for Chest Disease NYPH-CUMC, 622 W. 168th Street, New York, NY 10032, USA \\ ${ }^{2}$ Department of Biobehavioral Sciences, Teachers College, Columbia University, 522 W. 120th Street, New York, NY 10027, USA \\ ${ }^{3}$ Division of Cardiology, Department of Medicine, Columbia University Medical Center, 622 W. 168th Street, \\ New York, NY 10032, USA \\ ${ }^{4}$ Department of Physical Medicine and Rehabilitation, Montefiore Medical Center, 111 E. 210th Street, Bronx, NY 10467, USA \\ ${ }^{5}$ Center for Chest Disease, New York Presbyterian Hospital, 622 W. 168th Street, New York, NY 10032, USA
}

Correspondence should be addressed to Aimee M. Layton; am12135@columbia.edu

Received 15 August 2014; Revised 10 November 2014; Accepted 24 November 2014; Published 11 December 2014

Academic Editor: Manolis Tsiknakis

Copyright (C) 2014 Aimee M. Layton et al. This is an open access article distributed under the Creative Commons Attribution License, which permits unrestricted use, distribution, and reproduction in any medium, provided the original work is properly cited.

\begin{abstract}
The purpose of this study was to determine the feasibility and acceptability of utilizing a smartphone based application to monitor compliance in patients with cardiac disease around discharge. For 60 days after discharge, patients' medication compliance, physical activity, follow-up care, symptoms, and reading of education material were monitored daily with the application. 16 patients were enrolled in the study ( 12 males, 4 females, age $55 \pm 18$ years) during their hospital stay. Five participants were rehospitalized during the study and did not use the application once discharged. Seven participants completed 1-30 days and four patients completed $>31$ days. For those 11 patients, medication reminders were utilized 37\% (1-30-day group) and 53\% ( $>31$-day group) of the time, education material was read 44\% (1-30) and 53\% (>31) of the time, and physical activity was reported $25 \%(1-30)$ and $42 \%(>31)$ of the time. Findings demonstrated that patients with stable health utilized the application, even if only minimally. Patients with decreased breath sounds by physical exam and who reported their health as fair to poor on the day of discharge were less likely to utilize the application. Acceptability of the application to report health status varied among the stable patients.
\end{abstract}

\section{Introduction}

In order to improve healthcare quality for patients with several chronic conditions, the Patient Protection and Affordable Care Act of 2010 instituted penalties for hospital reimbursement if a patient admitted for myocardial infarction, congestive heart failure, or pneumonia was readmitted to the institution within 30 days of the original discharge (Patient Protection Affordable Care Act 2010). As such, hospitals have been faced with identifying patients in these cohorts who may be at high risk for hospital readmission and implementing interventions in hopes of improving patient care and reducing penalties that may be incurred by early readmission $[1,2]$.

Multicomponent interventions that feature early assessment of discharge needs have been found to be beneficial in reducing readmission rates [3]. These multicomponent interventions have made use of education, timely transfer to primary care teams, post-acute followup between 24 and $72 \mathrm{hrs}$ by nurse of physician, and appropriate referrals to support services such as rehabilitation programs [3] and 
the use of home exercise programs [4]. Although these programs may be effective, the extensive time and staffing needed for their implementation and success can limit their feasibility [5]. Alternatives to one-on-one patient to healthcare provider interaction have begun to be developed to potentially allow for a greater implementation of such interventions [6]. Various telemedicine approaches, such as smartphone applications, are being considered as potential tools for allowing healthcare professionals to implement and monitor patients remotely.

Smartphone applications are inexpensive and, unlike other forms of telemedicine, do not require home installation [7]. Smartphone applications can be utilized not just for accessing and tracking health information but also as a tool allowing practitioners and the patient's social support system to become more involved in his/her care without being physically present and in educational content delivery [8].

Areas where wireless health monitoring has been found to have succeeded in monitoring and tracking patients' health have been in patients with heart failure [9]. Patient's weight, blood pressure, and symptoms have been successfully monitored remotely by several telemedicine or structured telephone support systems [10]. However, these studies utilized multifaceted systems that provided and received information by more than just a smartphone application. Also the adoption of patients to utilize telemedicine systems has been an issue [11]. Chaudhry et al. found that recently discharged patients with heart failure had poor adherence of using the telemedicine system given to them after discharge. Given the age and demographics of heart failure patients, it is not clear whether a strictly smartphone application would be well adopted or demonstrate similar adoption problems as other telemedicine systems. Although there are potential benefits of utilizing smartphone technology for monitoring patients who are at high risk for being readmitted, the feasibility of monitoring heart failure patients or postmyocardial infarction patients via a strictly smartphone application has not yet been well documented. To our knowledge, there has been no report on the frequency of application use or potential barriers these patients may have in utilizing such technology after hospitalization.

The purpose of this study was to investigate the feasibility and acceptability of a smartphone iOS application to monitor and assist with patient medication compliance, education, home exercise, symptom changes, and transition to outpatient care team after hospitalization. The aim of this study was to determine the frequency of application use, potential barriers of use, and potential dropout rate in collecting this type of data in such population.

\section{Methods}

This study was a qualitative study discussing the homebased feasibility and acceptability of utilizing a smartphone application to collect health information and interact with patients with CHF or CAD around discharge.

The iOS application administered daily educational material, medication reminders, doctor appointment reminders, and monitored activity level. Process measurements, such as user engagement, daily task completion, and perceived value of the application to the patient, were recorded. Secondary outcome measures were activity level, medication compliance, follow-up care, enrollment into support programs such as cardiac or pulmonary rehabilitation program when applicable, and 30-60 day readmission rates in our population. Patients were given the usual standard of care throughout the study and the iOS application was only used to supplement outpatient discharge instructions and compliance, not to be used in place of the usual standard of care. Patients were instructed to follow all of their doctors' and nurses instructions and if there were any questions or confusion to contact their physician. All treating physicians were notified of their patient's participation in the study.

2.1. Participants. Hospitalized patients with a diagnosis of coronary artery disease (CAD) or congestive heart failure (CHF) were recruited. Patients were not considered for enrollment until clinical staff informed the study personnel that the patient would be eligible for discharge within the next 3 days. Study exclusion criteria consisted of a lack of described diagnosis, inability to perform physical activity, unstable angina, neurological deficit that makes the individual unable to understand and follow directions, being illiterate, nonEnglish-speaking, and no home WiFi connection.

Physicians and nurses working on the inpatient cardiac units identified 180 patients for the study team who were admitted with a primary diagnosis of CAD or CHF and were eligible for discharge. The study team then approached the patients and screened them for patient interest and eligibility. The study staff made it clear that participation was completely voluntary and all usual care would continue regardless of study participation. If the patient demonstrated interest in participation, the study staff informed the patient of what study participation would involve and received his/her consent to participate. This study was approved by the Columbia University Medical Center's Institutional Review Board. All participants signed informed consent prior to participating.

2.2. Protocol. Once patients consented to participating in the study, either the iOS application (Wellframe Application by Wellframe, Cambridge, MA) was uploaded to their smartphone or, if the patient did not own a device that could operate an iOS application, an iPod touch (iPod touch model A1367, Apple Inc., Cupertino, CA) was lent to the participant for the duration of the study. Patients were then given an orientation to how to use the device. A one-page instruction sheet was also given to the patient and the patient was instructed to practice using the application during their hospital stay. Study staff then followed up with the patient prior to discharge to ensure that there were no further questions regarding the use of the application. Figure 1 demonstrates the study personnel follow-up phone call protocol to collect information on rehospitalization, symptoms, and connection with outpatient care team.

Each day the participants received reminders to take their medication at self-selected times, brief condition 


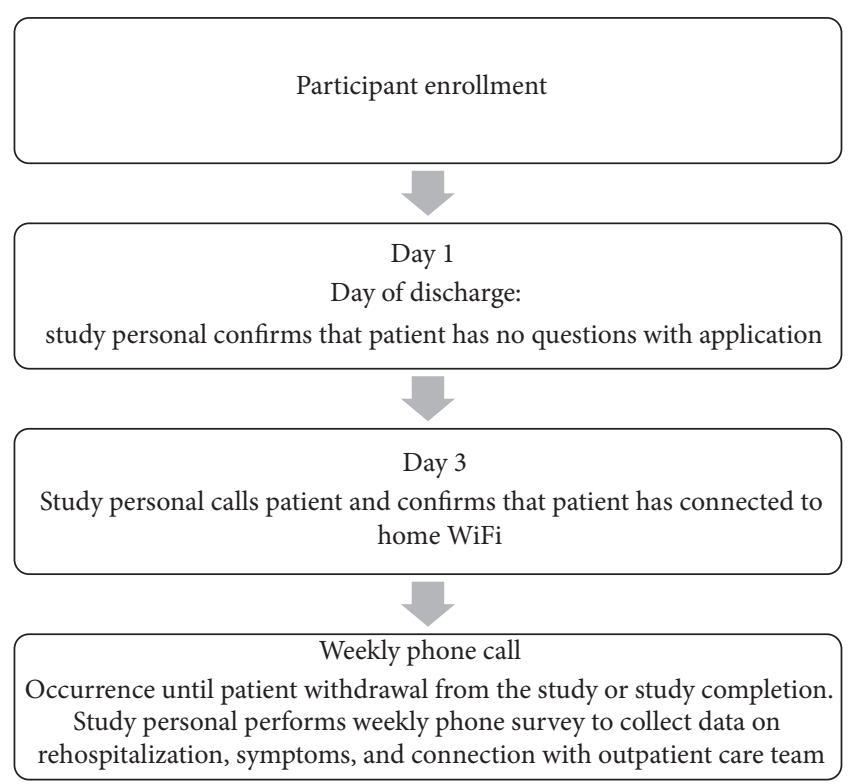

FIGURE 1: Study protocol.
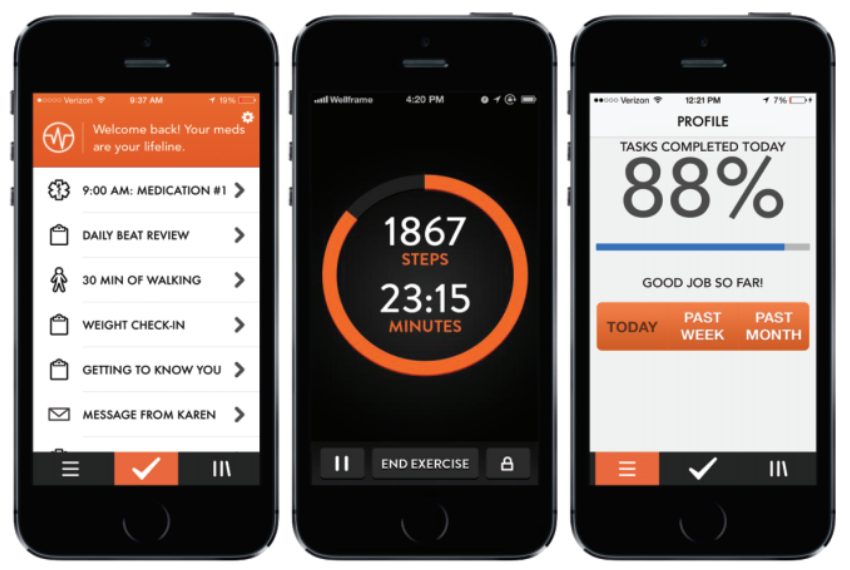

FIGURE 2: Example of application interface and content.

specific educational videos, and readings, and when relevant participants received reminders for upcoming appointments with healthcare providers. Additionally, participants could report changes in symptoms (e.g., dyspnea) and biometric measurements (e.g., heart rate) and track their physical activity through the iOS application's pedometer. Patients were considered "compliant" if greater than $50 \%$ of the "Daily To-Do Tasks" were completed. Figure 2 depicts an example of the application's "Daily To-Do Tasks."

\subsection{Measurements}

2.3.1. iOS Application Content. The Wellframe application recorded medication compliance by self-report. The time of day the patient takes his or her medication was programmed into the application. Each day, at the programmed time, a medication reminder would appear on the patient's smart phone or iPod touch. The patient would then have to confirm
TABLE 1: Educational content topics.

\begin{tabular}{ll}
\hline Topic & $\begin{array}{l}\text { General description of the following was } \\
\text { included: }\end{array}$ \\
\hline $\begin{array}{l}\text { Coronary heart } \\
\text { disease }\end{array}$ & Pathology, management, and symptoms \\
\hline Medications & $\begin{array}{l}\text { The value of medications, instructions for } \\
\text { taking medications, and potential } \\
\text { side-effects }\end{array}$ \\
\hline Exercise & $\begin{array}{l}\text { The benefits of exercise and guidance for } \\
\text { exercising safely }\end{array}$ \\
\hline Nutrition & $\begin{array}{l}\text { General nutritional recommendations } \\
\text { and benefits }\end{array}$ \\
\hline Smoking & $\begin{array}{l}\text { Benefits of smoking cessation/reduction } \\
\text { and resources for quitting }\end{array}$ \\
\hline Alcohol & $\begin{array}{l}\text { General guidance and recommendations } \\
\text { around alcohol consumption for } \\
\text { cardiovascular health and recovery }\end{array}$ \\
\hline Psychosocial & $\begin{array}{l}\text { Information and resources around mood } \\
\text { disorders }\end{array}$ \\
\hline $\begin{array}{l}\text { Biometric risk } \\
\text { factors }\end{array}$ & $\begin{array}{l}\text { Supporting patients to "know their } \\
\text { numbers" (ie., blood pressure, } \\
\text { weight/BMI, lipids, or oxygen when } \\
\text { applicable) }\end{array}$ \\
\hline
\end{tabular}

by touching "yes" or "no" if he or she took their medication. If the patient selected "no," an option to be reminded again in an hour would appear. If the patient selected "yes," then a second reminder would appear on the phone or iPod touch an hour later.

Educational material was given to the patient daily via the patient's "Daily To-Do's." The patient would be prompted to click on the educational reading material or video. Once the patient viewed the educational material, a check would appear on his or her "To-Do List" to demonstrate that task had been completed. The educational material consisted of disease management, smoking sensation, importance of attending cardiac or pulmonary rehab programs, and potential psychosocial issues associated with heart disease. Every day the patient received one of the "education topics" to read. Full list of topics can be seen in Table 1.

The patients were also sent daily messages encouraging him or her to perform daily walking, stretches, and light strengthening exercises (home based cardiac rehabilitation program) $[12,13]$.

When the patient tapped the prompt on his/her "ToDo" list to perform his/her daily exercises, he/she was then taken to a second screen that included instructions for the exercises, videos for the stretches and light strengthening exercises, and a pedometer for walking. At the end of the exercise regimen, the patient was asked to enter his/her level of breathlessness based on a modified Borg scale of perceived exertion [14]. For safety, the research team monitored the patient's exercises remotely and if a patient reported a level of breathlessness higher than 4 (somewhat severe), reported a heart rate exceeding $150 \mathrm{bpm}$, or had any adverse symptoms (e.g., dizziness, nausea, headache, chest discomfort, lightheadedness, drop in blood pressure, unusual heartbeat, 
or palpitations) the Medical Director of the Cardiac and Pulmonary Rehabilitation Program here at New York Presbyterian Hospital was alerted and a plan was in place to contact the patient. Patients were instructed to forgo the exercise program if they began a cardiac rehabilitation program.

Daily survey questions were asked regarding breathlessness, overall control of health, ease of using the application, medication side effects, and biometric measurements. Biometric measurements included weight, blood pressure, and heart rate.

During the weekly phone calls, the study staff would ask the patient if he or she had been enrolled in a cardiac rehabilitation program or had any upcoming doctor appointments. If the patient responded in the affirmative, then the staff would ask for the dates and times and enter the appointments into the patient's application via the dashboard. Staff also surveyed the patient to if he or she found the application useful and how is it most helpful. Lastly, the staff would inquire if the patient has seen his/her physician during the past week and if he or she has been recently hospitalized (if yes, then why). If the patient could not be reached after three attempts (over the course of 3 days), then a person the patient had designated as an acceptable emergency contact person for the study was contacted to confirm the patient's safety and if he or she had been hospitalized.

2.3.2. The Dashboard. Patient data was uploaded to a remote dashboard for the study staff to view and send messages to the patient via a secure server (approved by Columbia University Information Technology Department as a HIPPA compliant and secure server approval forms attached). Data was uploaded real time and the study staff would contact the patient via the application 1-2 times per week to provide encouragement. The study team could also request a response from a patient via the messaging system on the dashboard and modify a patient's medication reminders or exercise protocol if necessary via the dashboard or by contacting Wellframe.

2.3.3. Chart Review. Demographics, anthropometry, and ejection fraction and information about the patient's hospital stay were obtained by reviewing the electronic hospital chart. Information about any complications during the patient's stay, breath sounds by physical exam, and medications were retrieved from the physicians and/or nursing notes. For "day of discharge" information, notes within $24 \mathrm{hrs}$ of discharged were considered acceptable and data from that note were retrieved. The pain scale and resting dyspnea scores were collected from the physical therapist's note on the day of discharge or the day prior to discharge if there was no physical therapy note provided at the day of discharge. Sleep quality and self-report description of healthy values were obtained via the Wellframe application at the day of discharge. All patients were asked to respond to the "daily beat questions" on the day of discharge so that the study team could ensure that the application was working properly.

2.4. Data Analysis. We tested the feasibility of using this application in patients with $\mathrm{CHF}$ or CAD after hospitalization by collecting data on the frequency of application use, usage barriers described by the patient population, study dropout rate, and type of data patients were more or less likely to provide.

Based on previous behavior usage research, investigating the usage of smartphone applications [15], we selected to analyze standard ethnographic and user data. Application usage was described by analyzing the frequency of response to symptom and biometric data survey questions, medication reminders, clicks on educational content, pedometer readings, and clicks of stretches and strengthening exercise videos. The logging in of system usage to detect adherence has been utilized in other telemedicine studies [11, 16, 17]. Study population size is similar to previous feasibility studies [17, 18]. Acceptability was tested by collecting data on application usage and types of questions patients choose to respond to about their health. To better define the type of patients that may be more or less likely to utilize the application, objective health parameters and self-described health status were collected. Patient's responses and usage were graphed to determine behavioral trends. Statistical analysis was performed using SPSS version 21 (IBM Corp., Armonk, NY, 10504). Nonnumeric variables (insurance, dyspnea, breath sounds, and description of health) were coded by severity. A linear regression test was used to determine whether any of the variables collected had a relationship with the amount with which a patient utilized the application. Significance was set a priori at $P<0.05$.

\section{Results}

3.1. Participant Characteristics. Between July 2013 and December 2013, 158 patients were approached for the study. Twenty-two patients who were identified as eligible for the study were not approached because they were asleep or with a treating physician when study personnel attempted recruitment. In all of these occurrences the study personnel attempted to return to the patient's room for recruitment; however, the patient had been discharged. Figure 3 depicts the breakdown of patients approached, declined participation, met exclusion criteria, and included. Of the 158 patients approached, 16 were enrolled in the study (12 M, 4F, age $55 \pm 18$ years). Of the 16 patients, two were African American, one was Asian, one was Hispanic, and twelve were Caucasian. The largest barriers to enrollment were language and access to home WiFi.

Table 2 describes each patient's demographics, diagnosis, and socioeconomic status based on insurance provider. Ten of the patients who participated in the study had a diagnosis of CAD and were hospitalized for a cardiac intervention. Five of the patients were hospitalized with a primary admitting diagnosis of CHF. Most of our patients had private insurance providers.

3.2. Acceptability Analysis. The number of days patients interacted with the application after discharge and the characteristics of the patient's health are described in Table 3. The five patients who were readmitted to the hospital during 


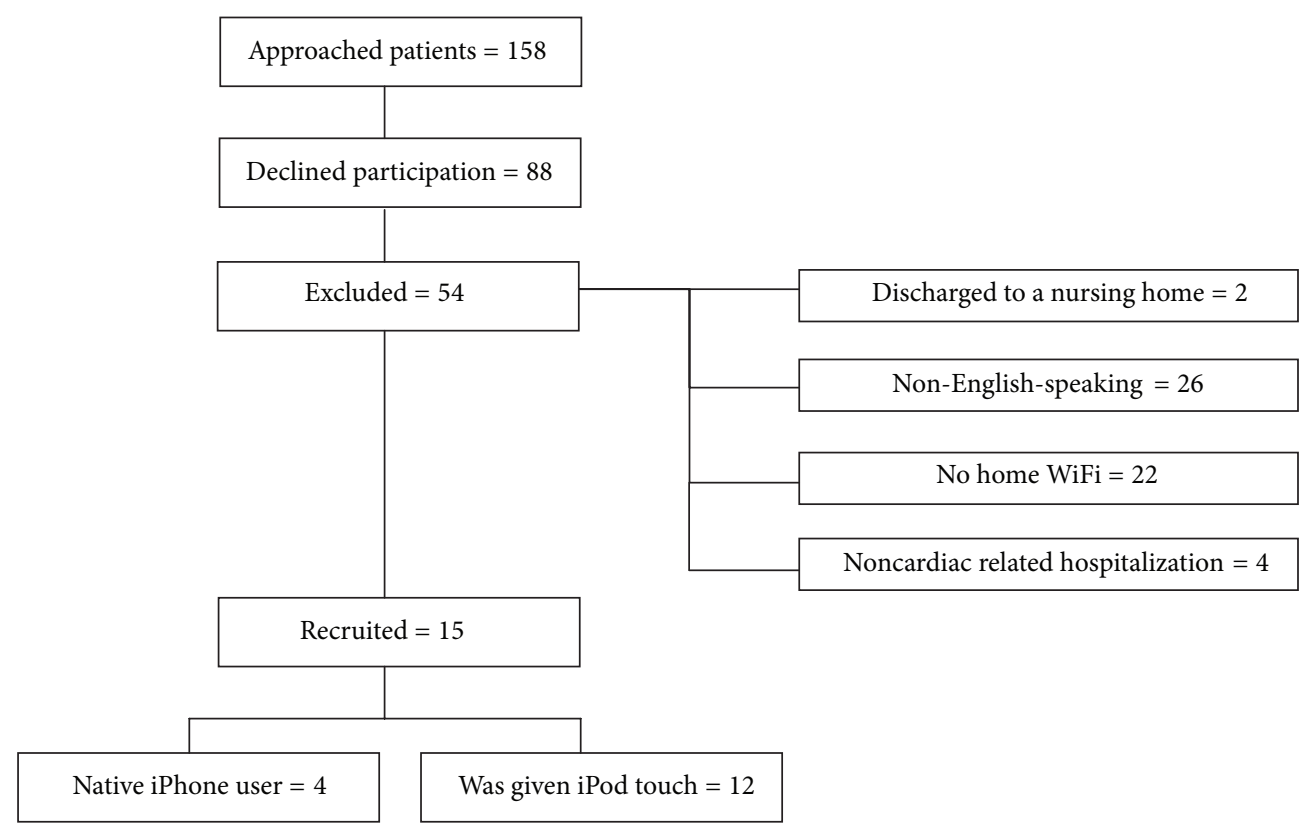

FIGURE 3: Participant recruitment and study population diagram.

TABLE 2: Demographics.

\begin{tabular}{lcccc}
\hline Subject & Age & Dx & Gender & Socioeconomic status by insurance provider \\
\hline 1 & 54 & CAD & M & Private Ins \\
2 & 50 & CHF & Medicare \\
3 & 84 & CAD & M & Medicare \\
4 & 40 & CHF & M & None \\
5 & 81 & CHF & M & Private Ins \\
6 & 49 & CHF & F & Medicaid \\
7 & 37 & CAD & M & Private Ins \\
8 & 40 & CAD & M & Private Ins \\
9 & 69 & CAD & M & Private Ins \\
10 & 60 & CAD & F & Private Ins \\
11 & 26 & CAD & M & Medicare \\
12 & 72 & CAD & M & Medicaid \\
13 & 28 & CHF & M & Medicare \\
14 & 70 & CAD & M & Medicaid \\
15 & 71 & CAD & CHF & Private Ins
\end{tabular}

\begin{tabular}{|c|c|c|c|c|}
\hline Avg & 55 & $\mathrm{CAD}=10, \mathrm{CHF}=6$ & $M=12, F=4$ & $7=$ Private Ins, $4=$ Medicare, $3=$ Medicaid, $1=$ none \\
\hline
\end{tabular}

Dx: diagnosis, CAD: coronary artery disease, CHF: congestive heart failure, BMI: body mass index, Private Ins: private insurance company provider, EF: ejection fraction, M: male, and F: female.

the study duration (average of 7 days after discharge) did not utilize the application once discharged from the hospital. Of the remaining patients, all who were not readmitted, the application was utilized between 1 day and the complete 60 days. The average amount of days with which a patient utilized the application was 17 . The average body mass index revealed that our patients were on average obese. The reported estimated ejection fractions by ECHO (EF) (taken after intervention when applicable) revealed that the majority of our patients had an EF of $>55 \%$ except for 4 patients who had EFs of $<45 \%$. Of note, the patients who did not interact with the application were also the patients with more severe complications and complaints during their hospital stay, with the exception of one. This may reflect that sicker patients were less likely to utilize the application.

During the study, five patients (31\%) were readmitted to the hospital, with the average readmission time of 7 days. Of note, all five patients only interacted with the application while being in the hospital and did not interact with the application once discharged. Of the 11 other patients, who were not 
TABLE 3: Days interacted with the application after discharge, anthropometric measurement, severity of heart disease measurements, and hospital stay information.

\begin{tabular}{|c|c|c|c|c|c|c|c|}
\hline Subject & $\begin{array}{l}\text { Number of days patient } \\
\text { interacted with app after } \\
\text { discharge }\end{array}$ & $\mathrm{EF}$ & BMI $\left(\mathrm{kg} / \mathrm{m}^{2}\right)$ & $\begin{array}{l}\text { Length of } \\
\text { hospital stay }\end{array}$ & \multicolumn{2}{|c|}{$\begin{array}{l}\text { Readmitted to hospital } \\
\text { within } 60 \text { days }\end{array}$} & $\begin{array}{l}\text { Complications or } \\
\text { complaints post-op }\end{array}$ \\
\hline 1 & 0 & $65 \%$ & 28.9 & 19 & SOB & 7 days & $\begin{array}{l}\text { Afib, } \mathrm{R} \text { diaphragm } \\
\text { paralysis }\end{array}$ \\
\hline 2 & 0 & $20 \%$ & 39.4 & 6 & Infection & $24 \mathrm{hrs}$ & $\begin{array}{l}\text { Day of discharge, } \\
\text { complained of chills }\end{array}$ \\
\hline 3 & 0 & $65 \%$ & 29.4 & 19 & $\begin{array}{l}\text { Thrombo- } \\
\text { cytopenia }\end{array}$ & 3 days & $\begin{array}{l}\text { Volume overload, } \\
\text { Afib, knee pain }\end{array}$ \\
\hline 4 & 0 & $60 \%$ & 54.0 & 8 & $\begin{array}{l}\text { SOB chest } \\
\text { pressure }\end{array}$ & 3 days & SOB \\
\hline 5 & 0 & $10 \%$ & 23.8 & 3 & Cardiac arrest & $\sim 3$ wks & None \\
\hline 6 & 1 & $63 \%$ & 29.1 & 2 & \multicolumn{2}{|c|}{ No } & None \\
\hline 7 & 1 & $25 \%$ & 44.6 & 12 & \multicolumn{2}{|c|}{ No } & None \\
\hline 8 & 1 & $55 \%$ & 21.0 & 5 & \multicolumn{2}{|c|}{ No } & L foot pain \\
\hline 9 & 5 & $60 \%$ & 26.4 & 5 & \multicolumn{2}{|c|}{ No } & None \\
\hline 10 & 13 & $55 \%$ & 24.8 & 4 & \multicolumn{2}{|c|}{ No } & None \\
\hline 11 & 26 & $55 \%$ & 21.3 & 5 & \multicolumn{2}{|c|}{ No } & Post-op anemia \\
\hline 12 & 30 & $60 \%$ & 38.4 & 9 & \multicolumn{2}{|c|}{ No } & Back pain \\
\hline 13 & 40 & $55 \%$ & 25.0 & 8 & \multicolumn{2}{|c|}{ No } & None \\
\hline 14 & 48 & $60 \%$ & 27.1 & 9 & \multicolumn{2}{|c|}{ No } & None \\
\hline 15 & 52 & $55 \%$ & 27.4 & 13 & \multicolumn{2}{|c|}{ No } & Post-op anemia \\
\hline 16 & 58 & $45 \%$ & 24.1 & 13 & \multicolumn{2}{|c|}{ No } & None \\
\hline Mean & 17 & $51 \%$ & 30.3 & 9 & \multirow{2}{*}{\multicolumn{2}{|c|}{$5=$ Yes, $11=$ No }} & \\
\hline Std & 21 & $17 \%$ & 9.2 & 5 & & & \\
\hline
\end{tabular}

App: application, SOB: shortness of breath, Afib: atrial fibrillation, R: right, L: left, post-op: postoperative, R/O: rule out, and std: standard deviation.

readmitted during the course of the study, 8 interacted with the application within the first day after discharge, 1 interacted with the application 3 days after discharge, and 2 interacted with the application greater than 1 week after discharge (Table 1). Ten patients withdrew from the study prior to day 60; however, of these ten patients, five agreed to participate in the 60-day follow-up survey to verify if he or she had been hospitalized within 60 days after discharge. Four of the ten patients declined any further participation in the study. Retrospective chart review confirmed that all four patients were not readmitted to the hospital within 60 days of discharge.

3.2.1. Correlates of Application Use. Results demonstrated that patients with unstable health after discharge had a lack of application use. All patients who were readmitted during the study did not utilize the application once discharged. These results are not suggesting a causational relationship but a correlative relationship, where application use may be a good indicator of overall health status.

Other correlatives of application use were breath sounds by physical exam within 24 hrs of discharge and the patient's self-report of health status at the day of discharge (Table 4). Analysis revealed that decreased breath sounds or crackles by physical exam within $24 \mathrm{hrs}$ of discharge had a significant relationship with application use $\left(R^{2}=0.370, P=0.021\right)$. The patient's self-report of his or her health status at the day of discharge also had a significant relationship with application use $\left(R^{2}=0.335, P=0.038\right)$. Breath sounds and self-report of health were independent predictors of application use and could explain $79 \%$ of the variability in application use when modeled together $\left(R^{2}=0.625, P=0.02\right)$.

3.2.2. Usage Barriers. The patients that found the application helpful also utilized the application the most and remained in the study the longest. Reasons patients gave to why the application was not helpful were the inability to change medication reminder times, the inability to enter doctor's appointments or other reminders themselves (the study team would have to enter these reminders into the dashboard and then the patient would receive the reminder), the pedometer would stop counting if another application on the device was opened (this is a limitation of iOS software design), and the general inconvenience of being asked to use the application on a regular basis. Upgrades to the application software and user interface have resolved many of these issues. Unfortunately, the inconvenience of entering data for a research study is a common limitation to participant compliance. Positive responses to application use were the usefulness of the medication reminders, the educational information provided in the "Daily Beat," and the stretching and exercise videos.

3.3. Feasibility Analysis. Seven patients completed 1-30 days of the trial. Figure 4 demonstrates the overall compliance 
TABLE 4: Day of discharge information.

\begin{tabular}{|c|c|c|c|c|c|c|}
\hline Subject & Pain scale & Resting HR & Dyspnea & BS on exam DOD & $\begin{array}{l}\text { Day of discharge } \\
\text { described health } \\
\text { as }\end{array}$ & $\begin{array}{l}\text { Discharged with } \\
\text { pain medication }\end{array}$ \\
\hline 1 & 0 & 107 & Moderate & Decreased, wheeze & Fair & $\begin{array}{l}\text { Oxycodone, } \\
\text { Tylenol }\end{array}$ \\
\hline 2 & 5 & 99 & Mild & Decreased & Fair & Tylenol \\
\hline 3 & 0 & 74 & Moderate & Decreased/crackles & Good & $\begin{array}{l}\text { Oxycodone, } \\
\text { Percocet }\end{array}$ \\
\hline 4 & 0 & 85 & Very mild & N/A & Poor & None \\
\hline 5 & 0 & 93 & None & Crackles & Good & Aspirin \\
\hline 6 & N/A & 96 & None & Clear & Fair & Toradol, Tylenol \\
\hline 7 & 0 & 72 & Mild & Decreased & N/A & None \\
\hline 8 & 5 & 95 & None & Clear & N/A & Hydromorphone \\
\hline 9 & 0.5 & 71 & None & Trace crackles & Good & Aspirin \\
\hline 10 & 2 & 88 & None & Clear & N/A & $\begin{array}{c}\text { Oxycodone, } \\
\text { Tylenol }\end{array}$ \\
\hline 11 & 6 & 94 & Moderate & Clear & Good & Tylenol w/Codeine \\
\hline 12 & 10 & 68 & Moderate & Trace crackles & Fair & Aspirin, Vicodin \\
\hline 13 & 0 & 99 & None & N/A & Excellent & Aspirin \\
\hline 14 & 4 & 86 & Very mild & Clear & Good & Tylenol \\
\hline 15 & 0 & 100 & Very mild & Clear & Good & Oxycodone \\
\hline 16 & 0 & 100 & None & Clear & Very good & Tylenol, Morphine \\
\hline Mean & 2 & 89 & & & & \\
\hline Std & 3 & 12 & & & & \\
\hline
\end{tabular}

HR: heart rate, BS: breath sounds, DOD: day of discharge, std: standard deviation.

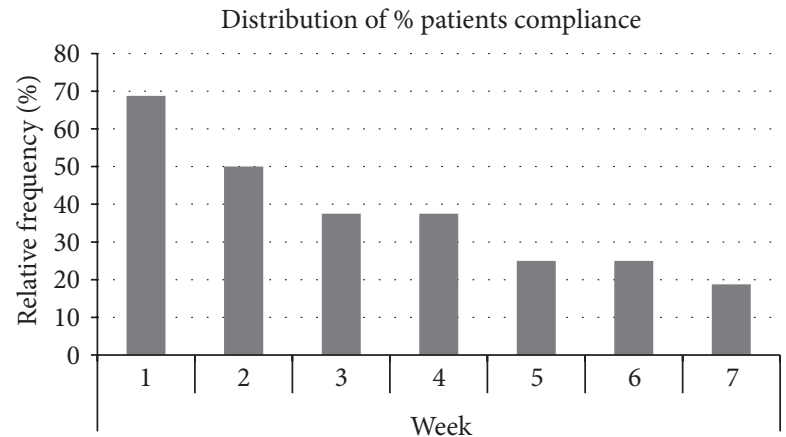

(a) Distribution of overall patient compliance

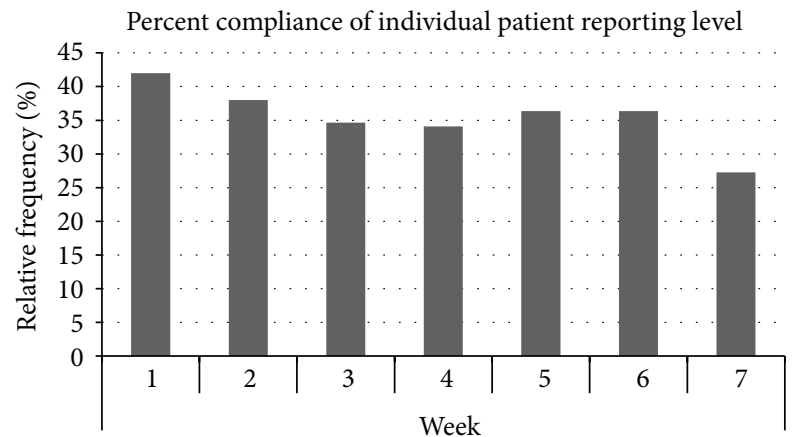

(b) Distribution of individual patient compliance

FIGURE 4: Distribution of compliance frequency.

of application usage. Sixty-nine percent of the patient population utilized the application during week one; however, the percent of relative frequency of use diminished to only $19 \%$ by week 7 , demonstrating poor adherence over time (Figure 4(a)). During week 1, the patients answered the application questions with a median of $42 \%$ of the time. Over time, compliance diminished to a nadir of $27 \%$ (Figure 4(b)). On average, those patients utilized the medication reminders $37 \%$ of the time, read education material $44 \%$ of the time, answered survey questions through the application $55 \%$ of the time, and performed the recommended physical activity $25 \%$ of the time. Four patients completed
31-60 days of the trial and utilized medication reminders $53 \%$ of the time, read education material $53 \%$ of the time, answered survey questions through the application $93 \%$ of the time, and performed the recommended physical activity $42 \%$ of the time. The data reveals that the patients who completed 31-60 days of the trial utilized the application more than those who withdrew prior to day 31. Patients who answered most of the survey questions continued to answer most of the survey questions throughout the study protocol. Patients who answered fewer than half of the survey questions in the first week proceeded to drop out to the study the following week. A common theme of patients who completed 
TABLE 5: Synopsis of survey questions and answers by patients after discharge.

\begin{tabular}{|c|c|c|c|c|c|c|c|}
\hline \multirow{2}{*}{ Survey questions } & \multicolumn{7}{|c|}{ Days after discharge } \\
\hline & $1-7(N=11)$ & $8-14(N=8)$ & $15-21(N=6)$ & $22-28(N=6)$ & $29-35(N=4)$ & $36-42(N=4)$ & $43-58(N=3)$ \\
\hline How breathless do you feel? & $54 \%$ & $75 \%$ & $100 \%$ & $83 \%$ & $100 \%$ & $100 \%$ & $100 \%$ \\
\hline Weight (lbs) & $50 \%$ & $83 \%$ & $40 \%$ & $50 \%$ & $100 \%$ & $100 \%$ & $100 \%$ \\
\hline $\begin{array}{l}\text { How easy do you find using } \\
\text { this app? }\end{array}$ & $22 \%$ & $33 \%$ & \multicolumn{5}{|c|}{ Question no longer asked } \\
\hline $\begin{array}{l}\text { Please count your pulse for } \\
20 \text { seconds }\end{array}$ & $31 \%$ & $63 \%$ & $83 \%$ & $67 \%$ & $100 \%$ & $100 \%$ & $100 \%$ \\
\hline $\begin{array}{l}\text { How would you describe } \\
\text { your health today? }\end{array}$ & $73 \%$ & $33 \%$ & $83 \%$ & $100 \%$ & $100 \%$ & $100 \%$ & $100 \%$ \\
\hline $\begin{array}{l}\text { I am in control of my own } \\
\text { health }\end{array}$ & $33 \%$ & $67 \%$ & $75 \%$ & $75 \%$ & $100 \%$ & $100 \%$ & $100 \%$ \\
\hline $\begin{array}{l}\text { Do you like the exercises on } \\
\text { the app? }\end{array}$ & $29 \%$ & $67 \%$ & \multicolumn{5}{|c|}{ Question no longer asked } \\
\hline
\end{tabular}

greater than 31 days was the presence of a family member during study recruitment. It appeared that when the patient's family support valued the patient utilizing the application, the patient was more likely to utilize the application for $>31$ days and be more compliant with the use of the application.

Both groups participated with the survey tool that requested the patient to rate his or her breathlessness, take his or her pulse, enter his or her weight, describe his or her health that day, and respond to the ease of using the application's feature (Table 4). The patients who remained in the study the longest were the most compliant in answering these survey questions. Patients were more likely to answer the questions about shortness of breath, entering their weight and describing their health, and least likely to answer the questions regarding the ease of using the application or how in control of their health they felt they were (Table 4). In the patients who withdrew prior to day 31 , they utilized the educational material the most and the physical activity aspect of the application the least. The patients who completed 3160 days of the trial utilized the medication reminders and educational material equally.

\section{Discussion}

4.1. Principal Findings. This study has shown that collecting information regarding postdischarge compliance and patients' health status via smartphone application in patients with heart failure or coronary artery disease may be feasible but not without limitation. Our results indicate that patients who were medically stable were more likely to utilize the application than patients who are unstable. Results also demonstrated that patients were more likely to respond to medication compliance questions, read education content, and respond to a few survey questions but not all and were less likely to report physical activity through the application (Table 5).

Acceptability of the application was low but similar to that of other studies. Min et al. [18] reported a smart phone application median compliance rating of $41 \%$ in their population of women undergoing treatment for breast cancer. Chaudhry et al. [11] reported a 90\% adherence in telemonitoring use in a population of patients with heart failure during the first week of the study but adherence decreased to $~ 55 \%$ by week 26. The low application compliance reported by this and other studies may reflect that although remote monitoring is convenient, the sample sizes needed for such research may be larger than those of other forms of survey tools or interventions. Therefore, future research in the field of remote monitoring may want to consider this low compliance rate when calculating desired sample sizes.

Compliance results also revealed higher acceptability rate in patients who remained in the study for greater than 31 days versus those who withdrew prior to 31 days. This relationship revealed a subset of patients who were more likely to utilize smart phone applications than others. Application usage is difficult to predict; however, work by Shin et al. [19] found that cellular network, time of day, and previous app use are all highly related to application usage. Hospitals that are seeking to utilize this kind of technology to track and interact with high risk patients may want to take into account these factors to optimize usage. Companies seeking to design applications for this use may also want to take into account these factors when designing their application.

Factors that impacted acceptability or barriers for usage were reported to be the inability to interact with the application as much as the individual required. As the patient's health and needs evolved, the application needed to be able to evolve. Also in patients whose health status was deteriorating, the application usage was decreased. These findings demonstrated a utility with smartphone applications to identify patients that need a care team to intervene rather than relying on technological remote monitoring. As highlighted by Pandor et al. [10], remote monitoring of patient's with $\mathrm{HF}$ health does not appear to impact the course of the patient's health unless monitoring dictates an action. Even when the remote monitoring dictates an action, study results have not always been positive [11]. Telemonitoring did not appear to provide a benefit over usual care when used to decrease rehospitalization [11]. Therefore, remote monitoring appears to be most useful in highlighting changes in behavior, rather than eliciting changes in outcomes. Thus, data collected by remote monitoring may be most useful for hospitals that are looking 
to allocate resources towards the patients who are most likely to be readmitted, rather than replacing usual care [11]. These results are not suggesting a causational relationship but an associative relationship between health status and application use. It is important to stress that smartphone applications may be most successful in helping to bridge the communication between care team and patient rather than be used to replace the need for one-to-one in-person interaction.

A novelty of this application to the growing "telemedicine" market was the immediate feedback mechanism via the application to a dashboard the clinician can log into, rather than other immediate feedback systems such as phone or video conference. Additionally, the telemedicine systems that involve home setup can cause a delay in information being received by the hospital. The average readmission time in our patient population was 7 days and mode was 3 days, and thus there was value in the ability to follow patients' status upon immediate discharge. For the above reasons, more of the remote monitoring market is moving to application use. The results from this study can help guide researchers, administrators, and companies in what aspect of the application appeared to be valued by the patient, such as medication reminder and educational content, and what aspects were underutilized, such as the pedometer and ability to communicate with the study team via the application.

A barrier to utilizing certain telemedicine devices discussed in prior research has been the barrier of training staff and patients on how to use the technology appropriately and in a timely fashion [20]. Some physicians expressed frustration with the time and level of technological sophistication needed to utilize certain technology [20]. A benefit to this particular application was the intuitive and simple user interface that took almost no training. However, this application was very limited in the information the clinician can gain from it. Perhaps future versions or application will include interfacing with diagnostic equipment that can provide a pulse rate, distinguish an arrhythmia, and allow for photographs to be uploaded to the dashboard. This would expand the use of the application beyond distinguishing the patient's general health state. However the cost-benefit ratio must be considered. Gurné et al. [21] described the incremental costs of using some telemedicine systems as being greater than the benefit in some settings. With each interface that an application builds, the cost increases. Also the ethics of attempting to replace in-person interaction with a multidisciplinary team in high risk patient populations, such as the one studied, should be considered [21]. However, the use of such applications as a tool to extend the reach of the care team, rather than a replacement, may allow for an inexpensive and manageable technique to identify patients who are in need of such in-person care.

There were limitations to this pilot study. Our patient population was not representative of the average patient here at New York Presbyterian as our initial version of the application was only in English. Since the completion of the study a Spanish version has been developed. Further research is needed to determine if these findings can be extrapolated to the Spanish speaking population. The WiFi requirement also limited recruitment. It is our suggestion that future work should consider a data plan that allows the patient to utilize the device with or without $\mathrm{WiFi}$, although this feature does add a further expense. Future work in a larger and more diverse population may be beneficial to confirm the relationships reported in these findings and allow for findings to be extrapolated to other patient populations. Lastly, the use of such application as a survey tool was useful however limited. A validated health questionnaire was not utilized for this study. This made the interpretation of the survey data limited. As a test of feasibility, the survey tool demonstrated that patients would respond to the daily questions and what questions the patients preferred; however, the applicability of these responses was limited.

\section{Conclusion}

This study demonstrated the feasibility and acceptability of utilizing an iOS application to monitor outpatient behavior in a group of patients considered to be at "high risk for readmission." Findings demonstrated that patients with stable health utilized the application more than patients with unstable health. The acceptability of the application varied greatly. There remains a need to better define aspects of smart phone applications that will result in optimal patient compliance; however, these results demonstrated that usage alone may be a useful tool to highlight patients in need of closer monitoring.

\section{Conflict of Interests}

No author has any conflict of interests to report.

\section{Acknowledgment}

Wellframe Inc. supplied the smart phone applications and iPod touches for this study.

\section{References}

[1] G. Giamouzis, A. Kalogeropoulos, V. Georgiopoulou et al., "Hospitalization epidemic in patients with heart failure: risk factors, risk prediction, knowledge gaps, and future directions," Journal of Cardiac Failure, vol. 17, no. 1, pp. 54-75, 2011.

[2] H. M. Krumholz, Y.-T. Chen, Y. Wang, V. Vaccarino, M. J. Radford, and R. I. Horwitz, "Predictors of readmission among elderly survivors of admission with heart failure," American Heart Journal, vol. 139, no. 1, pp. 72-77, 2000.

[3] I. A. Scott, "Preventing the rebound: improving care transition in hospital discharge processes," Australian Health Review, vol. 34, no. 4, pp. 445-451, 2010.

[4] D. Seidel, A. Cheung, E.-S. Suh, Y. Raste, M. Atakhorrami, and M. A. Spruit, "Physical inactivity and risk of hospitalisation for chronic obstructive pulmonary disease," International Journal of Tuberculosis and Lung Disease, vol. 16, no. 8, pp. 1015-1019, 2012.

[5] S. Manning, "Bridging the gap between hospital and home: A new model of care for reducing readmission rates in chronic heart failure," Journal of Cardiovascular Nursing, vol. 26, no. 5, pp. 368-376, 2011. 
[6] S. D. Anker, F. Koehler, and W. T. Abraham, "Telemedicine and remote management of patients with heart failure," The Lancet, vol. 378, no. 9792, pp. 731-739, 2011.

[7] N. M. Hjelm, "Benefits and drawbacks of telemedicine," Journal of Telemedicine and Telecare, vol. 11, no. 2, pp. 60-70, 2005.

[8] P. Klasnja and W. Pratt, "Healthcare in the pocket: mapping the space of mobile-phone health interventions," Journal of Biomedical Informatics, vol. 45, no. 1, pp. 184-198, 2012.

[9] M.-K. Suh, C.-A. Chen, J. Woodbridge et al., "A remote patient monitoring system for congestive heart failure," Journal of Medical Systems, vol. 35, no. 5, pp. 1165-1179, 2011.

[10] A. Pandor, T. Gomersall, J. W. Stevens et al., "Remote monitoring after recent hospital discharge in patients with heart failure: a systematic review and network meta-analysis," Heart, vol. 99, no. 23, pp. 1717-1726, 2013.

[11] S. I. Chaudhry, J. A. Mattera, J. P. Curtis et al., "Telemonitoring in patients with heart failure," The New England Journal of Medicine, vol. 363, no. 24, pp. 2301-2309, 2010.

[12] K. Jolly, R. S. Tayor, G. Y. H. Lip et al., "Home-based exercise rehabilitation in addition to specialist heart failure nurse care: design, rationale and recruitment to the Birmingham rehabilitation uptake maximisation study for patients with congestive heart failure (BRUM-CHF): a randomised controlled trial," BMC Cardiovascular Disorders, vol. 7, article 9, 2007.

[13] H. M. Dalal, P. H. Evans, J. L. Campbell et al., "Home-based versus hospital-based rehabilitation after myocardial infarction: a randomized trial with preference arms-Cornwall Heart Attack Rehabilitation Management Study (CHARMS)," International Journal of Cardiology, vol. 119, no. 2, pp. 202-211, 2007.

[14] G. Borg, "Ratings of perceived exertion and heart rates during short-term cycle exercise and their use in a new cycling srength test," International Journal of Sports Medicine, vol. 3, no. 3, pp. 153-158, 1982.

[15] T. M. T. Do, J. Blom, and D. Gatica-Perez, "Smartphone usage in the wild: a large-scale analysis of applications and context," in Proceedings of the ACM International Conference on Multimodal Interaction (ICMI '11), pp. 353-360, November 2011.

[16] D. Scherr, P. Kastner, A. Kollmann et al., "Effect of home-based telemonitoring using mobile phone technology on the outcome of heart failure patients after an episode of acute decompensation: randomized controlled trial," Journal of Medical Internet Research, vol. 11, no. 3, p. e34, 2009.

[17] S. Winkler, M. Schieber, S. Lücke et al., "A new telemonitoring system intended for chronic heart failure patients using mobile telephone technology-feasibility study," International Journal of Cardiology, vol. 153, no. 1, pp. 55-58, 2011.

[18] Y. H. Min, J. W. Lee, Y. W. Shin et al., "Daily collection of selfreporting sleep disturbance data via a smartphone app in breast cancer patients receiving chemotherapy: a feasibility study," Journal of Medical Internet Research, vol. 16, no. 5, p. e135, 2014.

[19] C. Shin, J. H. Hong, and A. K. Dey, "Understanding and prediction of mobile application usage for smart phones," in Proceedings of the 14th International Conference on Ubiquitous Computing (UbiComp '12), pp. 173-182, September 2012.

[20] D. L. Paul, K. E. Pearlson, and R. R. McDaniel Jr., "Assessing technological barriers to telemedicine: technologymanagement implications," IEEE Transactions on Engineering Management, vol. 46, no. 3, pp. 279-288, 1999.

[21] O. Gurné, V. Conraads, L. Missault et al., "A critical review on telemonitoring in heart failure," Acta Cardiologica, vol. 67, no. 4, pp. 439-444, 2012. 

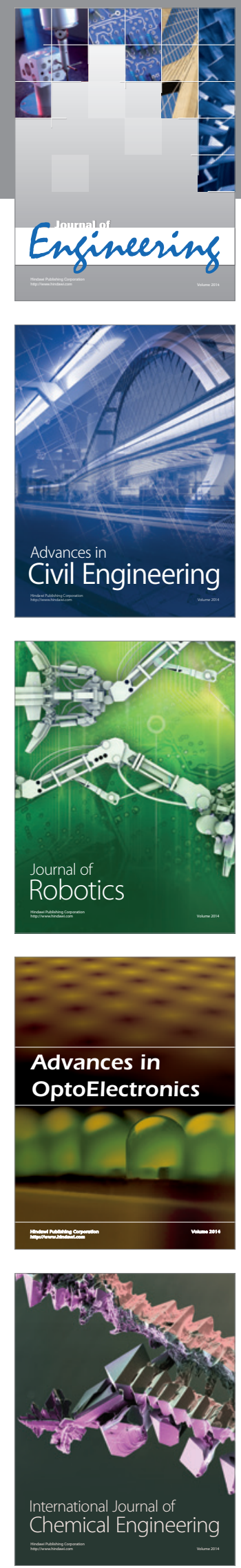

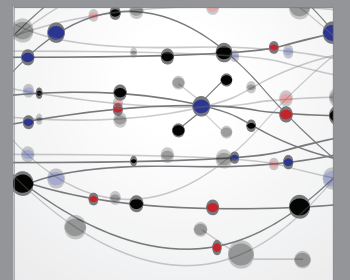

The Scientific World Journal
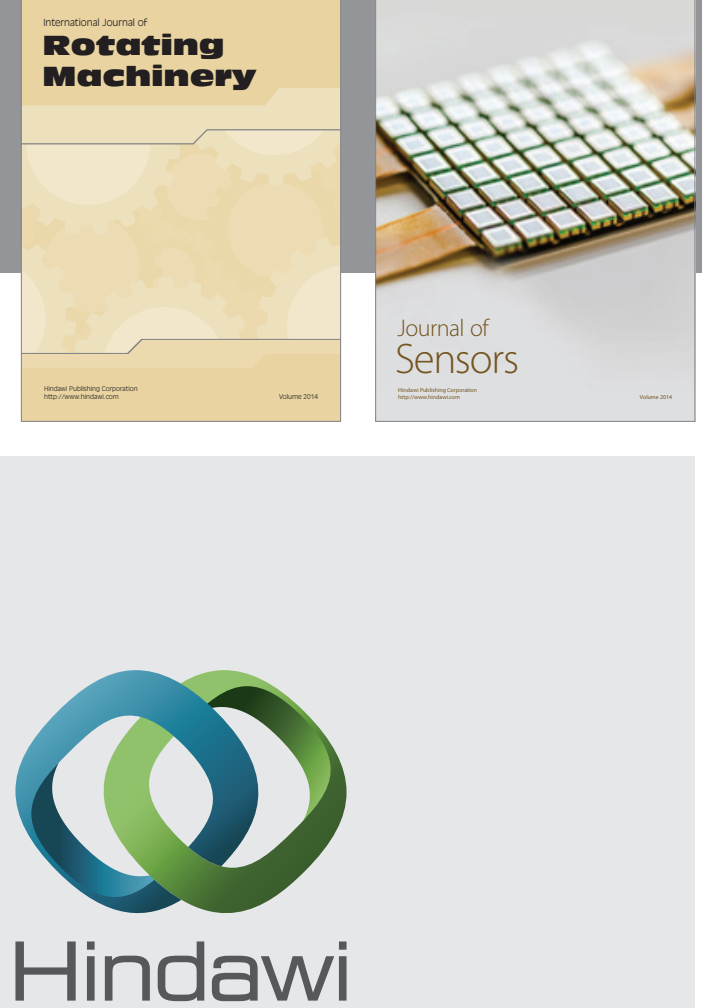

Submit your manuscripts at http://www.hindawi.com
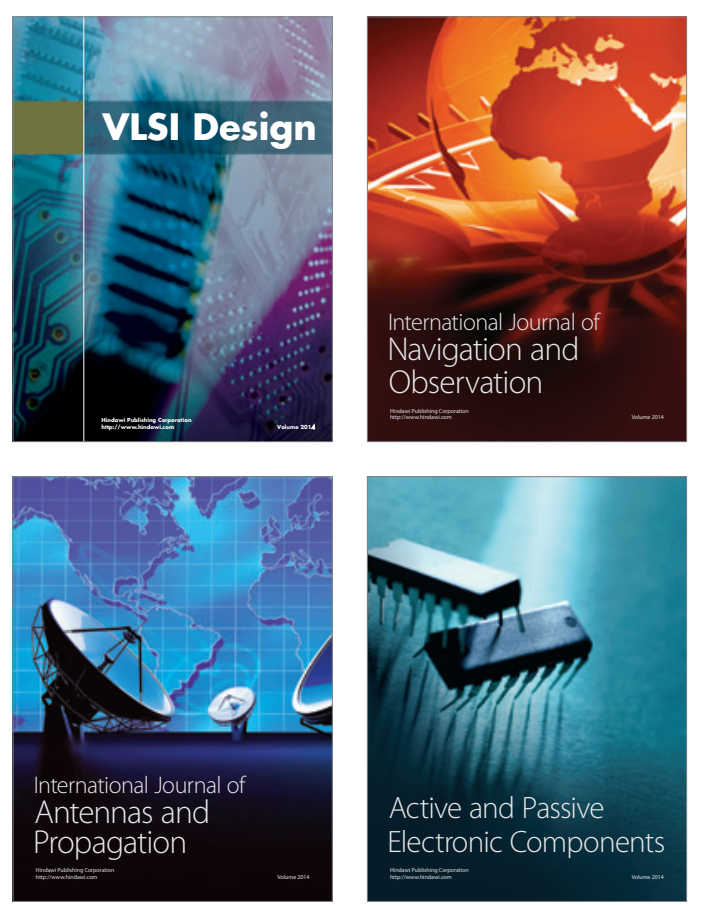
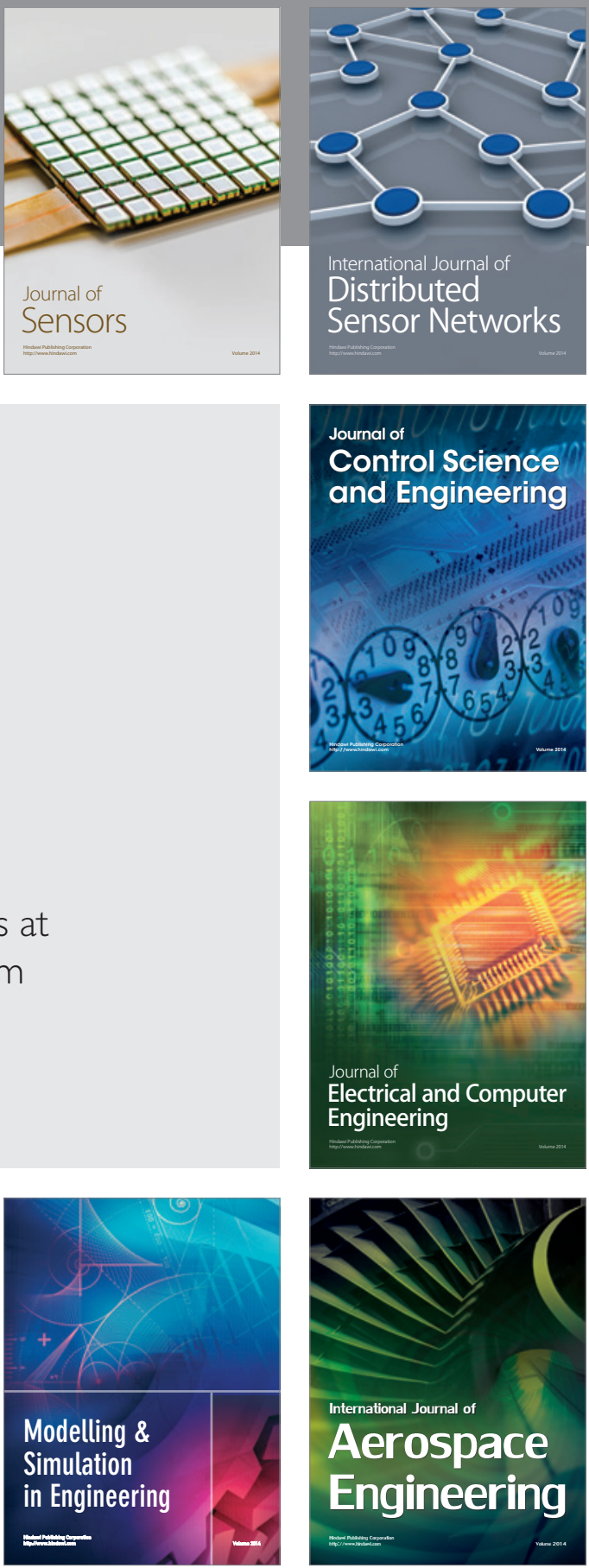

Journal of

Control Science

and Engineering
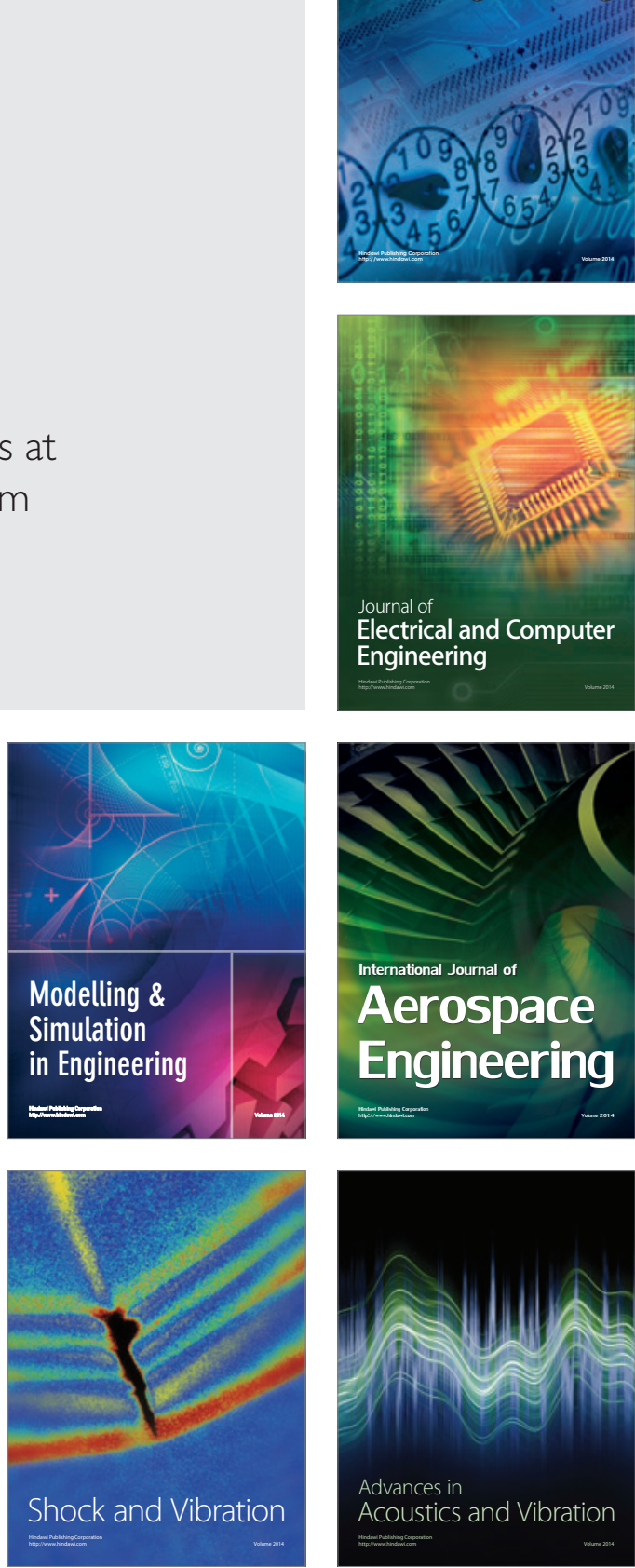